\title{
One-way and near-absolute polarization insensitive near-perfect absorption by using an all-dielectric metasurface
}

\author{
Deniz Umut Yildirim, ${ }^{1,2, *}$ (1) Amir Ghobadi, ${ }^{1,2}$ (1) Mahmut Can Soydan, ${ }^{1,2}$ (1) \\ Andriy E. SerebryanniKov, ${ }^{1,3,4}$ and EKmel Ozbay ${ }^{1,2,5,6,7}$ \\ ${ }^{1}$ NANOTAM-Nanotechnology Research Center, Bilkent University, 06800, Ankara, Turkey \\ ${ }^{2}$ Department of Electrical and Electronics Engineering, Bilkent University, 06800, Ankara, Turkey \\ ${ }^{3}$ Faculty of Physics, Adam Mickiewicz University, 61-614 Poznan, Poland \\ ${ }^{4}$ Department of Electrical and Electronics Engineering, Universidad Publica de Navarra, 31006 Pamplona, Spain \\ ${ }^{5}$ Department of Physics, Bilkent University, 06800, Ankara, Turkey \\ ${ }^{6}$ UNAM-Institute of Materials Science and Nanotechnology, Bilkent University, Ankara, Turkey \\ ${ }^{7}$ e-mail: ozbay@bilkent.edu.tr \\ ${ }^{*}$ Corresponding author: yildirim@ee.bilkent.edu.tr
}

Received 8 January 2020; revised 28 February 2020; accepted 29 February 2020; posted 2 March 2020 (Doc. ID 387350); published 30 March 2020

In this Letter, we numerically propose the one-way perfect absorption of near-infrared radiation in a tunable spectral range with high transmission in the neighboring spectral ranges. This functionality is obtained by using a two-dimensional, guided-mode resonance-based grating-waveguide metasurface that acts as a frequencyselective reflector, a spacer dielectric, and an absorbing oxide layer. Within the bandwidth of the excited guidedmode resonance excited at $1.82 \mu \mathrm{m}$ (with a full-width at half-maximum of $19 \mathrm{~nm}$ ), we confirmed perfect absorption when light was incident from one of the two opposite directions, whereas in the other direction, perfect reflection was observed. The forward-to-backward absorption ratio reached as high as 60 , while the thickness of the entire structure was on the order of the operating wavelength. In addition to the spectral tunability of the excited resonances and their bandwidths, our proposed device supports transparency windows with $65 \%$ transmission in the adjacent frequency bands. Our 2D grating is also verified to enable near-absolute insensitivity to the polarization state of incident light. Geometrical parameter modification also gives our design great tunability, as we also designed a device with a $300 \mathrm{~nm}$ absorption/reflection linewidth. () 2020 Optical Society of America

https://doi.org/10.1364/OL.387350

Confinement and absorption of electromagnetic radiation is one of the most vigorously studied research areas under metamaterials. While narrowband absorbers are of specific interest in the areas of sensing, imaging, and color filtering [1-3], their broadband analogs [4] are promising in the areas of thermal photovoltaics [5], hot-electron-based photodetectors [6], and passive radiative cooling [7]. Some applications, however, may require multifunctional devices, i.e., having different functions at a specific wavelength range, while having different functions in the adjacent bands $[8,9]$. A practical multifunctional device may be one yielding perfect, one-way electromagnetic wave (EMW) absorption in a required frequency band, while supporting transparency in the neighboring bands. In such a device, perfect absorption is to be achieved during resonance, while illumination from the opposite direction causes total reflection [10]. Although the widely used metal-insulator-metal (MIM) cavity architecture [11] may provide near-unity perfect absorption (reflection) in forward (backward) illumination, there are no adjacent transparency windows and no frequency selectivity due to the inherent characteristics of the optically thick bottom metal mirror.

Dielectric metasurfaces, comprising periodically arranged sub-wavelength inclusions, can be engineered to display the guided-mode resonance (GMR) phenomenon [12-14]. GMRs define the phase-matched coupling of free-space radiation to the supported (guided) modes of a waveguide, and the periodicity of the structure results in the leaky nature of these resonances. By utilizing these, frequency-selective perfect reflectors can be actualized with the desired bandwidth characteristics [15]. It is also desirable to have polarization insensitivity as a feature of these frequency-selective reflectors (FSRs), which is readily available in bulk metal bottom reflectors. While much work is focused on $1 \mathrm{D}$ grating structures that are inherently polarization sensitive [16], by focusing on $2 \mathrm{D}$ arrays, it is possible to have near-absolute polarization insensitivity.

In the present Letter, we use a $2 \mathrm{D}$ sub-wavelength gratingwaveguide structure to excite its GMRs, and subsequently use it as a near-absolute polarization insensitive FSR, on top of which there is a spacer dielectric and an absorbing indium tin oxide (ITO) layer. We employ the finite-difference time-domain (FDTD) method to numerically show that the coupling of 
incident light to the GMRs yields a resonance band, in which perfect absorption of near-infrared radiation is obtained under forward illumination (90\%) with $19 \mathrm{~nm}$ of full-width at halfmaximum (FWHM) at $1.82 \mu \mathrm{m}$. In the backward direction, the reflection reaches as high as $98.5 \%$, resulting in an ultra-high forward-to-backward reflection ratio of 60 . In the adjacent frequency bands, the transmission reaches as high as $65 \%$. We also show the tunability of the bandwidth of the resonant regions, as we also designed a device with a resonance bandwidth of $300 \mathrm{~nm}$.

Our proposed device in Fig. 1(a) is an FSR-insulator-oxide (MIO) cavity. The bottom FSR, which employs a unique 2D, sub-wavelength, periodically modulated, high permittivity germanium (Ge) grating, is used to redirect the otherwise transmitted EMWs back into the cavity. The spacer dielectric, $\mathrm{SiO}_{2}$, is used to create the necessary phase accumulation to redirect the EMWs mostly to the top absorbing layer of our cavity, which is a planar layer of ITO, a lossy plasmonic material. The entire structure is supported by a quartz substrate. It is important to mention that throughout this Letter, $\mathrm{SiO}_{2}$ perfectly covers the grating grooves and extends above them. Furthermore, forward (backward) excitation is defined as the case when EMWs are incident from the ITO (substrate) side, i.e., when EMWs propagate in the $-z(+z)$ direction, defined in Fig. 1(a). Figure 1(b) illustrates the unit cell of our 2D sub-wavelength grating FSR from the top view. In this device, the position of the resonant spectral region and its $Q$-factor are highly related to the thickness of the grating region, $t_{\mathrm{Ge}}$; periodicity, $P$, of the unit cell in $y$ direction; and width of each grating stripe, $w$. The effects of all of these factors on GMRs and fabrication complexity are investigated in detail in our recent works $[1,15]$. The cavity resonance condition due to the accumulated phase in a round-trip in the cavity, and the strength of absorption in both forward and backward cases are determined by $t_{\mathrm{SiO}_{2}}$ and $t_{\mathrm{TTO}}$, respectively.
We initiate our analysis by first scrutinizing the excitation of GMRs in the FSR, which defines the frequency band where there is the forward absorption/backward reflection functionality. To investigate the spectral response of device I, we utilize FDTD simulations. In the simulations, we illuminated the unit cell with a broadband plane wave at normal incidence whose electric field was polarized in the $x$ direction $(\phi=0, p$ polarization), as in Fig. 1(a). In the $x$ and $y$ directions of the simulation region, we employed periodic boundary conditions, while in the $z$ direction, perfectly matched layers (PMLs) were adopted. We used two monitors to find the reflected $(R)$ and transmitted $(T)$ power, normalized to the incident power. The dispersion effect of $\mathrm{Ge}, \mathrm{SiO}_{2}$, and ITO were retrieved with ellipsometry measurements. While the former two are in good agreement with the models based on the Sellmeier equation [17], permittivity of ITO is modeled by using the Drude-Lorentz model [15].

We first fixed $t_{\mathrm{Ge}}$ at $220 \mathrm{~nm}, P$ at $1100 \mathrm{~nm}, w$ at $240 \mathrm{~nm}$, and $t_{\mathrm{SiO}_{2}}$ at $1200 \mathrm{~nm}$, but had not yet incorporated ITO. We then looked at $R$ and $T$ of the FSR- $\mathrm{SiO}_{2}$ structure in the forward excitation case, to gain insight about resonance excitation. The simulation result in Fig. 1(c) shows a sharp GMR, manifesting itself as a peak in reflection (98\%) and a dip in transmission at $1.82 \mu \mathrm{m}$. The physics governing the excitation the GMRs, as well as the effect of FSR parameter on the GMR excitation wavelength and its bandwidth are particularized in our recent works $[1,15]$. Briefly, GMRs are excited when a diffracted order of the grating can excite a guided mode of the waveguide core, which is the FSR itself, as a result of phase matching [12], governed by Eq. (1):

$$
\begin{aligned}
k_{x, g} & =m K_{x}=n \frac{2 \pi}{\Lambda_{x}}=n \frac{2 \pi}{P \sqrt{3}}, \quad k_{y, g}=m K_{y}=l \frac{2 \pi}{\Lambda_{y}}=l \frac{2 \pi}{P}, \\
\beta & =\left|k_{x, g} \hat{\mathbf{x}}+k_{y, g} \hat{\mathbf{y}}\right|,
\end{aligned}
$$
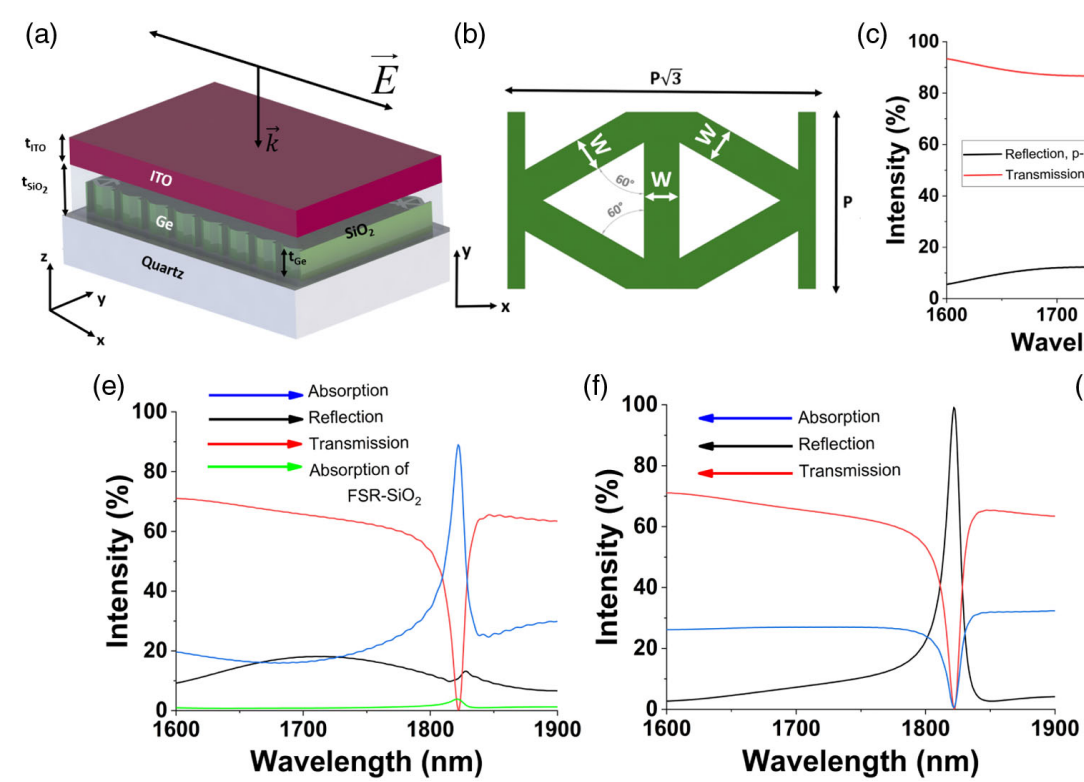

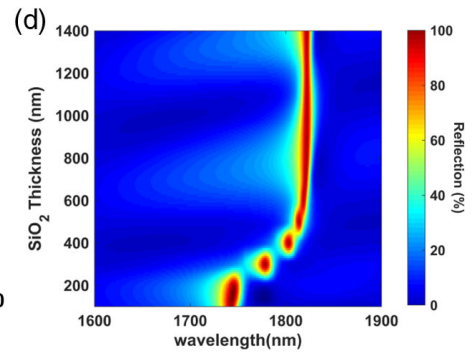

(g)

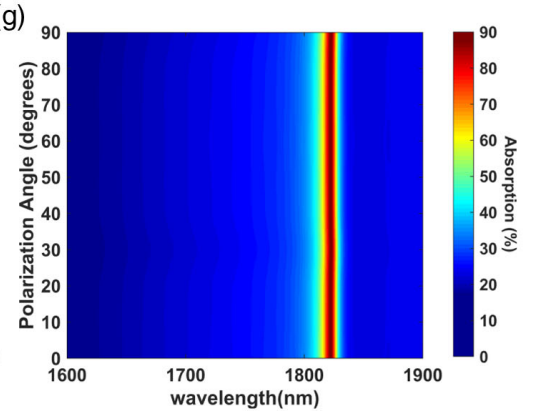

Fig. 1. Achieving one-way absorption/reflection with adjacent transparency windows in a narrowband design. (a) Proposed device to achieve one-way absorption/reflection with transparency in sideband functionality. $\mathrm{SiO}_{2}$ layer is drawn transparently to make the FSR visible as well. (b) Unit cell of FSR, elaborating the 2D grating, as seen from the top view. (c) Transmission and reflection spectra of the FSR - SiO ${ }_{2}$ structure under $p$ polarized incident plane wave. (d) Spectral response of the FSR - $\mathrm{SiO}_{2}$ structure when thickness of the $\mathrm{SiO}_{2}$ spacer, $t_{\mathrm{SiO}_{2}}$, is changed. (e) Reflection map of FSR $-\mathrm{SiO}_{2}$ structure for the polarization angles spanning from $0^{\circ}$ ( $p$, TM polarization) to $90^{\circ}(s$, TE polarization). Spectral response of the narrowband device under (f) forward and (g) backward illumination. 
where $\beta$ is the propagation wavenumber along the guiding direction, $\Lambda_{x}\left(K_{x}\right)$ and $\Lambda_{y}\left(K_{y}\right)$ are the grating periods (reciprocal lattice vectors) along $x$ and $y$ directions, respectively, $m(l)$ is the diffraction order along the $x(y)$ direction, and $k_{x, g}\left(k_{y, g}\right)$ is the wavevector component of the guided mode along the $x(y)$ direction. As the guided mode propagates, it also continually leaks energy to the far field because of the periodicity of the waveguide core. The sharp increase in reflection is because the re-radiated waves interfere destructively with the directly transmitted waves, but constructively with the reflected waves.

It is critical to mention that as long as $t_{\mathrm{SiO}_{2}}$ goes beyond the evanescent decay length of the guided mode in $\mathrm{SiO}_{2}$, it has little effect on the resonance wavelength, which is the case in our design. We verified this by keeping $t_{\mathrm{Ge}}, P$, and $w$ at their aforementioned values, and sweeping $t_{\mathrm{SiO}_{2}}$ from $100 \mathrm{~nm}$ to $1400 \mathrm{~nm}$, in which the lower bound only partially covered the grooves of the grating. The reflection spectrum shown in Fig. 1(d) demonstrates that $t_{\mathrm{SiO}_{2}}$ has a profound effect on the resonance when it is smaller than $t_{\mathrm{Ge}}$, because it controls the effective refractive index of FSR. For $t_{\mathrm{SiO}_{2}}$ values between $t_{\mathrm{Ge}}=220 \mathrm{~nm}$ and $400 \mathrm{~nm}$, the effective waveguide core refractive index is set, but the non-negligible optical fields in the $\mathrm{SiO}_{2}$-air boundary necessitate new boundary conditions and hence modify $\beta$. For $t_{\mathrm{SiO}_{2}}$ larger than $400 \mathrm{~nm}$, the resonance wavelength is indeed independent of $t_{\mathrm{SiO}_{2}}$.

Next, to obtain the desired functionality, we add the planar ITO layer of $t_{\mathrm{ITO}}=90 \mathrm{~nm}$ on the top $\mathrm{SiO}_{2}$ spacer to attain near-perfect EMW absorption for forward-propagating radiation. From this point on, $\rightarrow$ and $\leftarrow$ symbols will be used for absorption $(A), R$, and $T$ in forward and backward illumination cases, respectively. From $R$ and $T$, we calculated the spectral absorption by using $A=1-R-T$. The spectral response of our proposed narrowband device, under forward and backward illumination cases, is shown in Figs. 1(f) and $1(\mathrm{~g})$, respectively. These two figures confirm the multifunctionality of our device, as in the resonant range there is absorption (reflection) under forward (backward) illumination, while neighboring transparency windows with almost $65 \%$ transmittance are also obtained. With the values of $\vec{A}=0.9$ and $\overleftarrow{A}=0.015$ ( $\overleftarrow{R}=0.985$ ), the forward-to-backward absorption contrast, $\psi=\vec{A} / \overleftarrow{A}$, reaches as high as 60 , while the linewidth (FWHM) is measured as $19 \mathrm{~nm}$. The independence of the transmission from the illumination direction, i.e., having $\vec{T}=\overleftarrow{T}$, stems mainly from the Lorentz reciprocity [18] and energy conservation, while the mismatch in $\vec{A}$ and $\overleftarrow{A}$ originates from the fact that in the forward excitation case, the reflected EMWs are directed back to the ITO layer and harnessed mostly there, whereas in the backward case, they do not reach ITO and leave the device from the substrate. We also explored the contribution of FSR- $\mathrm{SiO}_{2}$ to the near-perfect absorption in the forward excitation case, by placing three $3 \mathrm{D}$ monitors on the $\mathrm{FSR}-\mathrm{SiO}_{2}$ layer. We used the formula in Eq. (2):

$$
\frac{d P_{\text {loss }}}{d V}=\frac{1}{2} w \epsilon^{\prime \prime}(w)|E|^{2},
$$

where $P_{\text {loss }}$ is the absorbed power due to conduction losses and dielectric hysteresis, $V$ is the volume, $w$ is the angular frequency, $\epsilon^{\prime \prime}(w)$ is the imaginary part of the complex permittivity, and $|E|^{2}$ is the modulus of the complex electric field inside each layer [19]. $d P_{\text {loss }} / d V$ is integrated over $\mathrm{FSR}-\mathrm{SiO}_{2}$ and normalized to the incident power to obtain absorption of it, which is plotted in Fig. 1(e). It is seen that during resonance, the FSR $-\mathrm{SiO}_{2}$ has an absorption of 0.04 , due to the small but non-negligible losses in Ge. ITO is then the main absorbing layer in our design.

We investigated the insensitivity to polarization in more detail by sweeping the polarization angle of the source from $0^{\circ}$ to $90^{\circ}$. The result shown in Fig. $1(\mathrm{~g})$ clearly indicates that our proposed device maintains almost constant absorption at a nearly fixed spectral position. This originates mainly from the fact that the hexagonal grating structure that we adopted has almost identical interaction with the incoming E-field for all polarization angles $[1,15]$.

To further discern the resonance phenomenon, we also plotted the electric and magnetic fields (E-field and H-field) profiles (a)

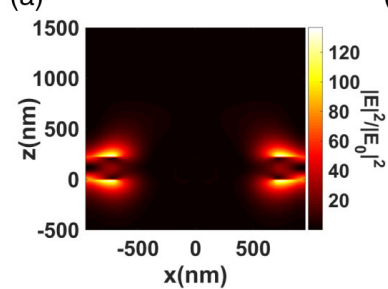

(e)

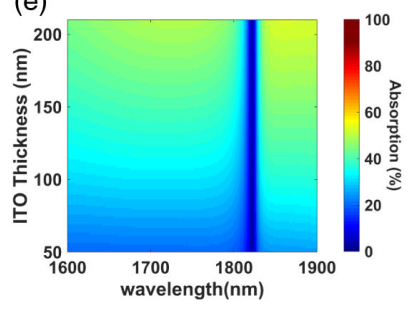

(b)

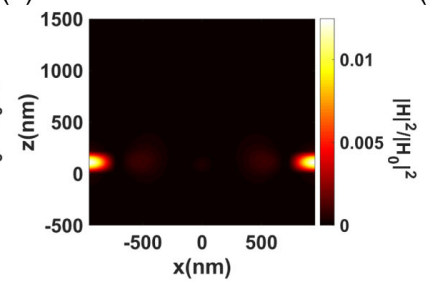

(f)

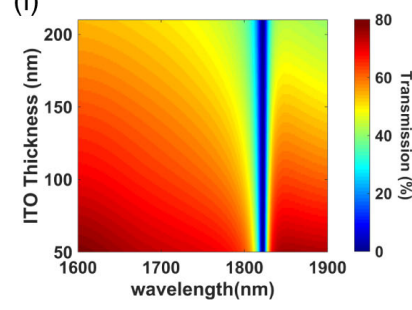

(c)

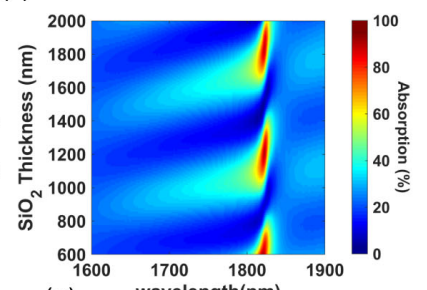

(g)

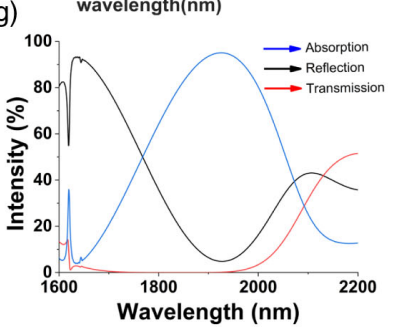

(d)

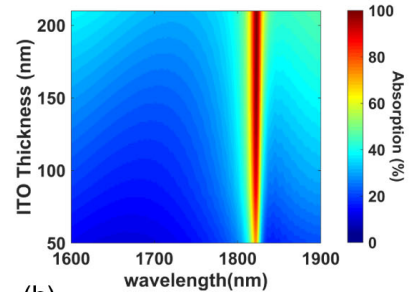

(h)

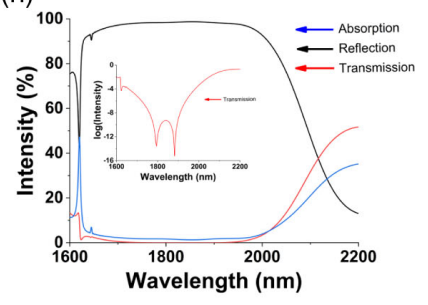

Fig. 2. (a) $|\mathrm{E}|^{2} /\left|\mathrm{E}_{0}\right|^{2}$ at $x z$ plane during resonance at $1.82 \mu \mathrm{m}$. (b) $|\mathrm{H}|^{2}$ at $x z$ plane during resonance. $|\mathrm{E}|^{2}$ and $|\mathrm{H}|^{2}$ are respectively the moduli of electric and magnetic fields inside device $\mathrm{I} ;\left|\mathrm{E}_{0}\right|^{2}$ and $\left|\mathrm{H}_{0}\right|^{2}$ denote those of incident light. GWS extends from $z=0$ to $z=220 \mathrm{~nm}$. Forward illumination absorption spectrum of the narrowband design under varying (c) $\mathrm{SiO}_{2}$ spacer thickness, $t_{\mathrm{Si}_{\mathrm{O}} \mathrm{N}_{2}}$; (d) ITO thickness, $t_{\mathrm{ITO}}$. Effect of $t_{\mathrm{ITO}}$ on the (e) absorption and (f) transmission spectra of the narrowband design under backward illumination. Spectral response of the broadband device under (g) forward and (h) backward illumination. 
at the peak resonance wavelength, in Fig. 2(a) and 2(b), respectively. These figures clearly indicate strong enhancement and localization of both fields inside the FSR region. These figures also imply that the fundamental mode of the slab waveguide is excited. This is an anticipated result because of the relatively small thickness of the waveguide core region with respect to the wavelength, $t_{\mathrm{Ge}} / \lambda$, as the excitation of higher-order modes of a waveguide requires greater core thickness.

In Fig. 2(c), we demonstrate how $t_{\mathrm{SiO}_{2}}$ affects $\vec{A}$, while all other geometrical parameters are fixed. $t_{\mathrm{SiO}_{2}}=1200 \mathrm{~nm}$ is picked because it is the smallest thickness that, first, yields the highest absorption, due to creating the necessary phase accumulation to redirect the EMWs mostly to the top absorbing layer. Second, it is much larger than the evanescent decay length of the guided mode, as shown in Fig. $1(\mathrm{~g})$, so it has no influence on the GMRs. Following this, we swept $t_{\mathrm{TTO}}$, and the result is shown in Fig. 2(d). Figure 2(d) indicates that the thicker layers of ITO boost $\vec{A}$. This is because EMWs travel more in ITO and are harvested before leaving the device. The choice of $90 \mathrm{~nm}$ is made by considering backward illumination and the absorption and transmission spectra in this case, as plotted with respect to $t_{\mathrm{ITO}}$ in Figs. 2(e) and 2(f), respectively. While $t_{\mathrm{ITO}}$ has little effect on $\overleftarrow{A}$ in the resonant band, as can be observed in Fig. 2(e), it decreases $\overleftarrow{T}$ considerably, as in Fig. 2(f). Therefore, $t_{\mathrm{ITO}}$ is picked as $90 \mathrm{~nm}$ to have near-perfect $\vec{A}$ of 0.9 in the resonant band, while not accentuating the losses in the adjacent bands.

We also designed a device that operates in a wide spectral band [20], by altering the geometrical parameters to $t_{\mathrm{Ge}}=420 \mathrm{~nm}, P=1100 \mathrm{~nm}, w=180 \mathrm{~nm}, t_{\mathrm{SiO}_{2}}=800 \mathrm{~nm}$, and $t_{\mathrm{TTO}}=110 \mathrm{~nm}$. The simulation results for this device, under forward and backward illumination are presented in Figs. 2(g) and 2(h), respectively. While $\overleftarrow{R}$ remained above 0.9 for a broad spectral range of $400 \mathrm{~nm}, \vec{R}$ did not show a flat perfect absorption, although the peak absorption reached 0.95 , and the linewidth was measured to be $300 \mathrm{~nm}$. To have a broad GMR bandwidth, the main design parameter that we changed was the thickness of the grating (FSR), $t_{\mathrm{Ge}}$. By doing so, the waveguide was able to support higher-order modes [19] in addition to the fundamental mode, and the overlap of these resulted in a broad spectrum. The coexistence of two modes is observed upon plotting the natural logarithm of transmission, as in the inset of Fig. 2(h). In this device, since there are two modes that are spectrally close and overlap with each other, the field profiles that correspond to distinct modes are superposed and, therefore, could not be plotted.

In conclusion, in this Letter, we analyze and numerically confirm one-way absorption/reflection with transmission in the sideband functionality of an all-dielectric metasurface. Near-absolute insensitivity of the proposed design to the polarization incident light is verified. Our 2D sub-wavelength periodically modulated grating-waveguide structure supports GMRs. Within the bandwidth of the resonance, it effectively acts as a mirror to create a Fabry-Perot-type cavity, due to the $\mathrm{SiO}_{2}$ spacer dielectric and the top layer ITO. In the narrowband design, the linewidth is calculated to be $19 \mathrm{~nm}$, while the forward-to-backward absorption ratio is found to be 60 . These results are complemented with $65 \%$ transparency in the sideband frequencies. A broadband design is also analyzed, and in the forward direction, resonant absorption with a peak strength of $95 \%$ and $300 \mathrm{~nm}$ linewidth is realized, while in the backward direction, reflection stays above 0.9 for $400 \mathrm{~nm}$. Overall, our simple yet robust, and easy-to-fabricate device is promising not only for applications in the near-infrared range but also for $\mathrm{THz}$ applications, such as obtaining single-frequency signals from $\mathrm{THz}$ sources, in which the multifunctionality shown in this Letter is required.

Funding. Türkiye Bilimsel ve Teknolojik Araştirma Kurumu (113E331, 114E374, 115F560); Türkiye Bilimler Akademisi.

Disclosures. The authors declare no competing financial or non-financial interests.

\section{REFERENCES}

1. D. U. Yildirim, A. Ghobadi, and E. Ozbay, Sci. Rep. 8, 15210 (2018).

2. C. Ji, K. T. Lee, T. Xu, J. Zhou, H. J. Park, and L. J. Guo, Adv. Opt. Mater. 5, 1700368 (2017).

3. A. Tittl, A. K. U. Michel, M. Schäferling, X. Yin, B. Gholipour, L. Cui, M. Wuttig, T. Taubner, F. Neubrech, and H. Giessen, Adv. Mater. 27, 4597 (2015).

4. M. C. Soydan, A. Ghobadi, D. U. Yildirim, V. B. Erturk, and E. Ozbay, Plasmonics 14, 1801 (2019).

5. J. K. Tong, W. C. Hsu, Y. Huang, S. V. Boriskina, and G. Chen, Sci. Rep. 5, 10661 (2015).

6. W. Li and J. Valentine, Nano Lett. 14, 3510 (2014).

7. D. U. Yildirim, A. Ghobadi, M. C. Soydan, O. Atesal, A. Toprak, M. D. Caliskan, and E. Ozbay, ACS Photon. 6, 1812 (2019).

8. T. Jang, H. Youn, Y. J. Shin, and L. J. Guo, ACS Photon. 1, 279 (2014).

9. M. Mutlu, S. Cakmakyapan, A. E. Serebryannikov, and E. Ozbay, Phys. Rev. B 87, 205123 (2013).

10. P. Rodríguez-Ulibarri, M. Beruete, and A. E. Serebryannikov, Phys. Rev. B 96, 155148 (2017).

11. K. Aydin, V. E. Ferry, R. M. Briggs, and H. A. Atwater, Nat. Commun. 2, 517 (2011).

12. D. Rosenblatt, A. Sharon, and A. A. Friesem, IEEE J. Quantum Electron. 33, 2038 (1997).

13. S. S. Wang and R. Magnusson, Appl. Opt. 32, 2606 (1993).

14. S. S. Wang, R. Magnusson, J. S. Bagby, and M. G. Moharam, J. Opt. Soc. Am. A 7, 1470 (1990).

15. D. U. Yildirim, A. Ghobadi, M. C. Soydan, M. Gokbayrak, A. Toprak, B. Butun, and E. Ozbay, J. Phys. Chem. C 123, 19125 (2019).

16. C. E. Rubio-Mercedes, V. F. Rodriguez-Esquerre, I. T. Lima, and H. E. Hernandez-Figueroa, J. Lightwave Technol. 32, 2163 (2014).

17. E. D. Palik, Handbook of Optical Constants of Solids (Academic, 2012).

18. R. J. Potton, Rep. Prog. Phys. 67, 717 (2004).

19. S. J. Orfanidis, Electromagnetic Waves and Antennas (2014).

20. W. Wu and R. Magnusson, Opt. Lett. 37, 2103 (2012). 